\title{
Estrutura fitossociológica de um fragmento natural de floresta inundável em área de Campo Sujo, Lagoa da Confusão, Tocantins
}

\author{
Elizabeth Rodrigues BRITOํ, Sebastiāo Venâncio MARTINS², Ary Teixeira de OLIVEIRA-FILHO², \\ Elias SILVA², Alexandre Francisco da SILVA ${ }^{4}$
}

\begin{abstract}
RESUMO
Fragmentos de florestas inundáveis localizam-se naturalmente na Planície do Araguaia entre os estados de Tocantins e Mato Grosso nas áreas de Cerrado. Ocorrem sob a forma de depressões naturais, que no estado do Tocantins estão situados nas áreas de planícies de inundação, que favorecem seu alagamento, conseqüentemente, o maior tempo de retenção da água em épocas de elevada precipitação. Este estudo teve como objetivo caracterizar a estrutura de um fragmento de floresta inundável de aproximadamente um hectare, inserido em área de Campo Sujo, no município de Lagoa da Confusão, TO. Foram amostrados todos os indivíduos arbustivo-arbóreos com circunferência 1,30m do solo (CAP) $\geq 15 \mathrm{~cm}$. Ao todo, foram encontrados 665 indivíduos, 34 famílias e 49 espécies. As espécies com maior valor de cobertura, em ordem decrescente, foram Sclerolobium paniculatum var. rubiginosum (Mart. ex Tul.) Benth., Calophyllum brasiliense Cambess. e Licania apetala (E. Mey.) Fritsch. As famílias mais ricas foram Fabaceae (8), Arecaceae, Chrysobalanaceae e Vochysiaceae (3). O índice de diversidade de Shannon $(\mathrm{H}$ ') foi de 2,97 nats/ind. A distribuição de classes de diâmetro apresentou curva na forma de "J" invertido estando a maioria dos indivíduos nas sete primeiras classes.
\end{abstract}

PALAVRAS-CHAVE: Floresta inundável, estrutura, classes de diâmetro, Savana.

\section{Phytosociological structure of a natural fragment of floodplain forest in area of Campo Sujo, municipal district of Lagoa da Confusão, Tocantins, Brazil.}

\begin{abstract}
Fragments of floodplain forests occur naturally in the Araguaia plain between the states of Tocantins and Mato Grosso on the ares of Cerrado. These fragments happen in form of natural depressions, which are located in areas of floodplains in the State of Tocantins, which favors its flooding and, consequently, the largest period of water retention during periods of high precipitation. The objective of this study was to characterize the structure of a fragment of floodplain forest of approximately one hectare, inserted in area of Campo Sujo, in Lagoa da Confusão, TO. Were sampled all the tree/shrub individuals with circumference at $1,30 \mathrm{~m}$ of the soil $(\mathrm{CAB}) \geq 15 \mathrm{~cm}$. On the whole, 665 individuals, 34 families and 49 species were found. The species with larger cover value, in descending order, were Sclerolobium paniculatum var. rubiginosum (Mart. former Tul.) Benth., Calophyllum brasiliense Cambess. and Licania apetala (E. Mey.) Fritsch.. The richest families were Fabaceae (8), Arecaceae, Chrysobalanaceae and Vochysiaceae (3). The Shannon diversity index ( $\left.\mathrm{H}^{\prime}\right)$ was 2,97 nats/ind. The distribution of diameter classes showed an inverted "J" curve.
\end{abstract}

KEY WORDS: Floodplain, structure, diameter classes, Savanna.

\footnotetext{
1 Instituto Natureza do Tocantins - Naturatins, Diretoria de Recursos Florestais, AANE 40, Ql 02, Lt. 3A, Alameda 1, 77054-080, Palmas - T0. Telefone: 63 3217.9056/63 3218.2613, email: erbrito@vicosa.ufv.br

2 Departamento de Engenharia Florestal, Universidade Federal de Viçosa, 36571-000, Viçosa - MG. Telefone: 31 3899.1187, email: venancio@ufv.br/ eshamir@ufv.br

${ }^{3}$ Departamento de Ciências Florestais, Universidade Federal de Lavras, 37200-000, Lavras - MG. Telefone: 35 3829.1413, email: aryfilho@ufla.br

${ }^{4}$ In memorian
} 


\section{INTRODUÇÃO}

A vegetação do Bioma Cerrado predominante no Brasil Central abrange cerca de 2 milhôes de $\mathrm{km}^{2}$ superado apenas pelo Bioma Amazônia que possui aproximadamente 3,5 milhôes de km² (Eiten, 1972; Nascimento \& Saddi, 1992; Ratter et al., 1997; Mendonça et al., 1998; Oliveira-Filho \& Fluminhan-Filho, 1999). Ocorre além do Brasil Central, em partes da região da Amazônia, do Nordeste, do Sudeste e em pequenas "ilhas" no Paraná (Felfili \& Silva Júnior, 1988; Oliveira-Filho \& Ratter, 1995; Ratter et al., 1997).

Entre os Estados de Tocantins e Mato Grosso, na Planície do Araguaia, predomina uma área de ecótono entre o Bioma Cerrado e Amazônia, que periodicamente sofre inundação na estação chuvosa durante quatro a cinco meses. Possui cerca de $600.000 \mathrm{~km}^{2}$ de extensão, tendo a maior representatividade na Ilha do Bananal. Essa região é composta por uma série de fisionomias, constituindo um mosaico vegetacional que inclui o Campo Limpo, o Campo Sujo, o Campo Cerrado, o Cerrado stricto sensu e o Cerradão, predominantes na paisagem, mais as inclusões florestais, como as florestas estacionais semideciduais aluviais e também um tipo vegetacional peculiar denominado regionalmente de "ipuca" ou "impuca" (Eiten, 1985).

As "ipucas" ou "impucas" são os fragmentos naturais de florestas inundáveis, que no Estado de Tocantins, surgem dentro das subformações da fisionomia campestre (campo sujo e/ou campo limpo) regionalmente denominados de "varjōes". Eiten (1985) denominou o "varjāo" como um tipo de pantanal caracterizado como um terreno não-florestado, anualmente encharcado ou rasamente inundado por quatro a cinco meses, de dezembro a março ou abril, e fortemente ressecado durante a estação seca (junho a setembro) e em vários locais com a presença de campos murundus. De acordo com Oliveira-Filho (1992) a origem dos murundus parece estar muito vinculada à atividade dos cupins, cujo solo formou-se a partir da construção dos ninhos pelos cupins e da erosão e degradação de numerosas gerações de cupinzeiros, em longo processo de sucessão.

As ipucas ocorrem em situações bem definidas, sob a forma de depressōes naturais que favorecem seu alagamento e conseqüentemente, maior tempo de retenção da água em épocas de maior precipitação, apresentando peculiaridades florísticas, estruturais e fisionômicas dos demais tipos vegetacionais do entorno, além de possuir a função de servir de abrigo, refúgio e também de fonte de alimentação para animais.

O presente estudo teve como objetivo analisar a estrutura fitossociológica das florestas inundáveis em virtude da expansão agropecuária e da crescente preocupação com a conservação dessas formaçôes vegetais.

\section{MATERIAL E MÉTODOS}

\section{ÁREA DE ESTUDO}

Este estudo foi realizado em um fragmento natural de floresta inundável no município de Lagoa da Confusão, Tocantins, numa propriedade rural denominada fazenda Lago Verde, situada entre as coordenadas UTM: 643586 a 655060 Leste e 8792795 a 8799167 Norte (Martins et al., 2002). A região está inserida na Bacia do Araguaia, que por sua vez possui largas extensōes de terras inundáveis. Trata-se de uma região de ecótono entre a Floresta Amazônica e o Cerrado (Martins et al., 2002), apresentando espécies representativas desses dois biomas. Essa área dista cerca de $50 \mathrm{~km}$ de duas Unidades de Conservação: Parque Nacional do Araguaia e Parque Indígena do Araguaia. A altitude está em torno de 180 $\mathrm{m}$, a temperatura média anual apresenta-se em torno de 24 ${ }^{\circ} \mathrm{C}$. O total médio anual de precipitação é de $1700 \mathrm{~mm}$, entre outubro e abril. O clima da região segundo a classificação de Köppen é do tipo Aw - tropical de verão úmido e período de estiagem no inverno (MILESKI, 1994).

Os solos da região apresentam manchas de Gleissolos e Plintossolos, são solos pouco profundos e estão sob forte influência do lençol freático (SEPLAN/TO, 2002). Na região, encontram-se fisionomias de cerrado, florestas de galerias e os fragmentos naturais de florestas inundáveis. Do ponto de vista sócio-econômico, essa região passa desde o finail dos anos 60 e, principalmente, nos anos 70 e 80 , por um intenso processo de ocupação por pecuaristas tradicionais e por grandes e pequenos produtores de grãos, favorecidos pela topografia plana propícia a mecanização.

\section{AMOSTRAGEM FITOSSOCIOLÓGICA}

Realizou-se a marcação numérica e identificação taxonômica de todos os indivíduos arbustivo-arbóreos com circunferência a $1,30 \mathrm{~m}$ do solo $(\mathrm{CAP}) \geq 15 \mathrm{~cm}$ em um fragmento de um hectare de área inserido em área de Campo Sujo. Foi coletado material botânico dos indivíduos amostrados para identificação, a qual foi realizada por comparação com material dos herbários da Universidade Federal de Viçosa, Universidade de Brasília e Universidade Federal de Lavras. Os nomes das espécies e seus respectivos autores foram confirmados e atualizados por bibliografia específica e também através do site do Missouri Botanical Garden (http://www.mobot.org/w3t/search/vast.html). O sistema de classificação adotado foi o APG II (2003).

Os parâmetros fitossociológicos calculados foram: densidade relativa, dominância relativa e a partir desses, o valor de cobertura (Mueller-Dombois \& Ellenberg, 1974). Foram calculados os índices de diversidade de Shannon $\left(H^{\prime}\right)$ e eqüabilidade (J') (Pielou, 1975) como indicadores de diversidade e heterogeneidade florística. Para calcular 
os referidos parâmetros foi utilizado o programa Fitopac 1 (Shepherd, 1996).

A distribuição de freqüência diamétrica foi realizada mediante o cômputo dos indivíduos amostrados de cada uma das espécies dentro da classe diamétrica a que pertencem (Harper, 1977). As classes de diâmetro foram estabelecidas com amplitude de $5 \mathrm{~cm}$, a partir do diâmetro mínimo de inclusão dos indivíduos.

\section{RESULTADOS E DISCUSSÃO}

\section{FITOSSOCIOLOGIA}

A flora arbustivo-arbórea inventariada no fragmento natural de floresta inundável resultou em 665 indivíduos, 34 famílias e 49 espécies. A densidade absoluta estimada foi de 665 indivíduos por hectare, área basal de $31,16 \mathrm{~m}^{2} / \mathrm{ha}$, diâmetro médio individual de $20,44 \mathrm{~cm}$, altura média de $9,21 \mathrm{~m}$. As famílias que mais contribuíram para a riqueza florística foram Fabaceae com oito espécies e Arecaceae, Chrysobalanaceae e Vochysiaceae com três espécies cada. As famílias que apresentaram apenas uma espécie representam $44 \%$ do total de indivíduos amostrados (Tabela 1).

De acordo com Oliveira-Filho \& Ratter (1995), a riqueza florística das florestas do Brasil Central pode ser atribuída, em parte, à forte contribuição de diferentes tipologias vegetacionais, particularmente das florestas Amazônica e Atlântica, do Cerrado e até mesmo da Caatinga. Porém, os fragmentos de florestas inundáveis estão condicionados ao encharcamento sazonal do solo e este influencia na germinação e no estabelecimento das espécies arbustivo-arbóreas.

A relação negativa entre umidade do solo e riqueza de espécies fica mais evidente quando se analisa gradientes de drenagem do solo em florestas ribeirinhas. Isso foi demonstrado numa floresta às margens de uma lagoa natural no Parque Estadual do Rio Doce, MG, em que nas parcelas sob alagamento periódico e, portanto, drenagem deficiente do solo, ocorreu baixa diversidade destacando-se em densidade espécies típicas desses ambientes como Xylopia emarginata Mart. e Calophyllum brasiliense (Camargos, 2004). O mesmo foi constatado num gradiente topográfico de floresta de várzea do estuário amazônico, em que os locais mais baixos com drenagem deficiente e baixos níveis de oxigênio por longos períodos, a diversidade de árvores foi muito baixa, com dominância ecológica de espécies de palmeiras (Cattanio et al., 2002).

A baixa riqueza de espécies arbóreas e o correspondente baixo valor do índice de diversidade de Shannon (H') (2,97 nats/ind.) podem ser atribuídos à seletividade de espécies que ocorre em florestas cujo solo encontra-se sob saturação hídrica. Essa baixa diversidade do componente arbóreo em conseqüência da inundação sazonal tem sido observada em florestas de várzeas no estuário amazônico (Cattanio et al., 2002) e de Porto Rico (Frangi \& Lugo, 1985) e em mata de galeria pantanosa de Nova Xavantina, MT (Felfili et al., 1988).

No Brasil Extra-Amazônico, tem sido encontrada também baixa diversidade de espécies nas florestas sob encharcamento permanente do solo, nas florestas higrófilas ou de brejo (Torres et al., 1994; Toniato et al., 1998; Ivanauskas et al., 1997; Paschoal \& Cavassan, 1999; Marques et al., 2003).

Verifica-se que seis espécies de seis famílias distintas somaram $55,34 \%$ do número total de indivíduos amostrados no fragmento estudado. As famílias mais abundantes foram: Fabaceae (Sclerolobium paniculatum var. rubiginosum, com 138 indivíduos); Clusiaceae (Calophyllum brasiliense, com 41 indivíduos); Chrysobalanaceae (Licania apetala, com 32 indivíduos); Ebenaceae (Diospyros guianensis, com 67 indivíduos), Rutaceae (Pilocarpus sp., com 42 indivíduos) e Annonaceae (Duguetia furfuracea, com 48 indivíduos).

As espécies amostradas com seus respectivos parâmetros fitossociológicos em ordem decrescente de valor de cobertura (VC) são apresentadas na Tabela 2. Sclerolobium paniculatum var. rubiginosum, a espécie com VC mais alto, exibiu a maior densidade e dominância relativas. De acordo com Felfili et al. (1999) essa espécie é comum no Cerradão e na Floresta Estacional e Semidecidual no Planalto Central. Na Amazônia, ocorre na vegetação secundária da Floresta Ombrófila Densa.

Calophyllum brasiliense apresentou o segundo maior valor de cobertura. Essa espécie está sempre condicionada à umidade do solo e tende a ocorrer nas áreas mais úmidas nas florestas de galerias como também tolera inundaçōes de forma que é comum nas várzeas amazônicas e está presente desde a América Central até o litoral norte catarinense (Oliveira-Filho \& Ratter, 1995; Oliveira-Filho \& Ratter, 2000; Camargos, 2004). Capaz de germinar após vários meses de submersão, embora, enquanto inundadas, as sementes não germinem, suas plântulas crescem normalmente, tanto em solo inundado quanto drenado, o que confere freqüência em vários ambientes ribeirinhos do Brasil (Scarano et al., 1997; Marques \& Joly, 2000). Esta espécie foi considerada como peculiar não-exclusiva de florestas de brejo com solo sob saturação hídrica (Ivanauskas et al., 1997). Em Nova Xavantina, MT, C. brasiliense foi considerada boa indicadora de mata de galeria pantanosa, ou seja, floresta que ocorre ao longo dos rios e cabeceiras em solos hidromórficos ricos em matéria orgânica (Felfili et al., 1998). Por essa seletividade a solos mal drenados, essa espécie tem sido indicada para a restauração de brejos e áreas ciliares alagáveis (Torres et al., 1992; Martins, 2001).

As demais espécies com maior VC destacaram-se por apresentar um considerável número de indivíduos como 
Licania apetala, Diospyros guianensis, Duguetia furfuracea, Himatanthus lancifolius, Vochysia divergens, Eschweilera ovata, Byrsonima intermedia, Mabea occidentalis.

Dentre as espécies ocorrentes na floresta estudada, Copaifera langsdorffii é considerada generalista por compartilhar áreas de florestas e o Cerrado, como outras são consideradas espécies seletivas de solos mais úmidos e com distribuição geográfica restrita ao Brasil Central, como Vochysia pyramidalis. Ocorreram também, espécies de locais propensos à inundação periódica de longa duração como Calophyllum brasiliense, Cecropia pachystachya e Vochysia divergens (Ivanauskas et al., 1997, Oliveira-Filho \& Ratter, 2000; Lobo \& Joly, 2000; Martins, 2001).

Do total de 665 indivíduos amostrados no fragmento natural de floresta inundável, apenas 59 (8,87\%) estão mortos, mostrando que não está ocorrendo intervenção antrópica que venha desencadear um índice de mortalidade expressivo.

Para o total de indivíduos, isto é, todas as árvores vivas, e para as cinco espécies com maior VC foram apresentadas as distribuições de indivíduos em classes de diâmetro nas Figura 1a a f: Sclerolobium paniculatum var. rubiginosum, Calophyllum brasiliense, Licania apetala, Diospyros guianensis e Duguetia furfuracea.

Houve predominância de maior densidade nas cinco menores classes de DAP $(77,90 \%)$, gerando um gráfico com a configuração de "J" invertido (Figura 1a). De acordo com Silva Júnior (1999), o padrão da curva em "J” invertido, com grande maioria dos indivíduos na primeira classe de diâmetro e a menor representação nas classes maiores, demonstra um balanço positivo entre o recrutamento e a mortalidade, o que caracteriza a mata como auto-regenerante. Esse padrão permite confirmar que existe uma distribuição equilibrada das diferentes classes de diâmetros, indicando um bom estado de conservação da comunidade estudada.

O padrão de distribuição dos indivíduos em classes de DAP, não se repetiu para todas as espécies com maior VC. Das espécies mais importantes, a maior concentração de indivíduos nas menores classes de DAP, foi encontrada apenas em Diospyros guianensis e Duguetia furfuracea (Figura 1e e f), sendo que apenas D. guianensis apresentou uma distribuição de classes de diâmetro aproximando-se de uma condição balanceada.

A maioria dos indivíduos de Licania apetala ocorreu nas classes intermediárias de diâmetros (Figura 1d), revelando a dificuldade da manutenção de regeneração natural da espécie nesse ambiente. Calophyllum brasiliense (Figura 1c) e Sclerolobium paniculatum var. rubiginosum (Figura 1b) apresentaram distribuiçóes irregulares dos indivíduos nas classes de DAP, o que pode estar refletindo maior mortalidade em determinadas classes e/ou falhas em eventos de frutificação/dispersão em determinados anos com reflexos na regeneração.

Apesar da seletividade de espécies, estes fragmentos naturais são extremamente importantes por conterem espécies adaptadas ao regime de alagamento do solo, representando as únicas manchas de vegetação arbórea dentro de uma paisagem campestre.

Portanto, tornam-se necessárias a adoção de medidas de proteção de áreas campestres da Bacia do Araguaia onde há ocorrência dessas florestas inundáveis, frente ao acelerado ritmo de desmatamento e degradação a que vem sendo submetidas.

Tabela 1 - Riqueza das famílias amostradas no fragmento natural de floresta inundável em área de Campo Sujo, Lagoa da Confusão, Tocantins.

\begin{tabular}{|c|c|}
\hline Famílias & Número de espécies \\
\hline FABACEAE & 8 \\
\hline ARECACEAE & 3 \\
\hline CHRYSOBALANACEAE & 3 \\
\hline VOCHYSIACEAE & 3 \\
\hline MALPIGHIACEAE & 2 \\
\hline RUTACEAE & 2 \\
\hline ANACARDIACEAE & 1 \\
\hline ANNONACEAE & 1 \\
\hline APOCYNACEAE & 1 \\
\hline AQUIFOLIACEAE & 1 \\
\hline BIGNONIACEAE & 1 \\
\hline CLUSIACEAE & 1 \\
\hline DILLENIACEAE & 1 \\
\hline EBENACEAE & 1 \\
\hline ELAEOCARPACEAE & 1 \\
\hline ERYTHROXYLACEAE & 1 \\
\hline EUPHORBIACEAE & 1 \\
\hline ICACINACEAE & 1 \\
\hline LACISTEMACEAE & 1 \\
\hline LAMIACEAE & 1 \\
\hline LAURACEAE & 1 \\
\hline LECYTHIDACEAE & 1 \\
\hline MELASTOMATACEAE & 1 \\
\hline MENISPERMACEAE & 1 \\
\hline MORACEAE & 1 \\
\hline MYRISTICACEAE & 1 \\
\hline MYRSINACEAE & 1 \\
\hline MYRTACEAE & 1 \\
\hline OCHNACEAE & 1 \\
\hline OLACACEAE & 1 \\
\hline PROTEACEAE & 1 \\
\hline RUBIACEAE & 1 \\
\hline URTICACEAE & 1 \\
\hline Indeterminada & 1 \\
\hline
\end{tabular}


ACTA

AMAZONICA

Estrutura fitossociológica de um fragmento natural de floresta

inundável em área de Campo Sujo, Lagoa da Confusão, Tocantins

Tabela 2 - Espécies amostradas no fragmento de floresta inundável e seus parâmetros fitossociológicos: $\mathrm{n}=$ número de indivíduos; $\mathrm{DR}=$ densidade relativa; $\mathrm{DoR}=$ dominância relativa; $\mathrm{VC}=$ valor de cobertura.

\begin{tabular}{|c|c|c|c|c|}
\hline Espécie & $\mathrm{n}$ & $\mathrm{DR}$ & DoR & VC \\
\hline Sclerolobium paniculatum var. rubiginosum (Mart. ex Tul.) Benth. & 138 & 20,75 & 21,94 & 42,69 \\
\hline Calophyllum brasiliense Cambess. & 41 & 6,17 & 12,89 & 19,05 \\
\hline Licania apetala (E. Mey.) Fritsch & 32 & 4,81 & 13,62 & 18,43 \\
\hline Diospyros guianensis (Aubl.) Gürke & 67 & 10,08 & 6,50 & 16,57 \\
\hline Pilocarpus sp. & 42 & 6,32 & 6,55 & 12,86 \\
\hline Duguetia furfuracea (A. St.-Hil.) Saff. & 48 & 7,22 & 2,90 & 10,12 \\
\hline Himatanthus lancifolius (Müll. Arg.) Woodson & 28 & 4,21 & 3,89 & 8,10 \\
\hline Vochysia divergens Pohl & 13 & 1,95 & 5,20 & 7,16 \\
\hline Eschweilera ovata (Cambess.) Miers & 21 & 3,16 & 2,68 & 5,84 \\
\hline Byrsonima intermedia A. Juss. & 23 & 3,46 & 1,45 & 4,91 \\
\hline Mabea occidentalis Benth. & 19 & 2,86 & 1,51 & 4,37 \\
\hline Swartzia macrostachya R.S. Cowan var. macrostachya & 9 & 1,35 & 1,86 & 3,22 \\
\hline Astrocaryum vulgare Mart. & 16 & 2,41 & 0,30 & 2,71 \\
\hline Curatella americana L. & 8 & 1,20 & 1,05 & 2,25 \\
\hline Sloanea garckeana K. Schum. & 2 & 0,30 & 1,90 & 2,21 \\
\hline Mauritiella armata (Mart.) Burret & 10 & 1,50 & 0,38 & 1,89 \\
\hline Cecropia pachystachya Trécul & 10 & 1,50 & 0,37 & 1,88 \\
\hline Erythroxylum anguifugum Mart. & 4 & 0,60 & 1,03 & 1,63 \\
\hline Tapirira obtusa (Benth.) D.J. Mitch. & 5 & 0,75 & 0,82 & 1,58 \\
\hline Calycorectes psidiiflorus (0. Berg) Sobral & 7 & 1,05 & 0,52 & 1,57 \\
\hline Dipteryx alata Vogel & 7 & 1,05 & 0,16 & 1,22 \\
\hline Emmotum nitens (Benth.) Miers & 4 & 0,60 & 0,59 & 1,19 \\
\hline Vochysia pyramidalis Mart. & 5 & 0,75 & 0,39 & 1,15 \\
\hline Sorocea guilleminiana Gaudich. & 5 & 0,75 & 0,13 & 0,88 \\
\hline Lacistema hass/erianum Chodat & 4 & 0,60 & 0,24 & 0,84 \\
\hline Guettarda viburnoides Cham. \& Schltdl. & 1 & 0,15 & 0,62 & 0,77 \\
\hline Heisteria laxiflora Engl. & 2 & 0,30 & 0,32 & 0,63 \\
\hline Plathymenia reticulata Benth. & 2 & 0,30 & 0,31 & 0,61 \\
\hline Hymenaea stigonocarpa Mart. ex Hayne & 1 & 0,15 & 0,45 & 0,60 \\
\hline Nectandra gardneri Meisn. & 3 & 0,45 & 0,13 & 0,58 \\
\hline Syagrus cocoides Mart. & 3 & 0,45 & 0,05 & 0,50 \\
\hline Hirtella glandulosa Spreng. & 2 & 0,30 & 0,19 & 0,49 \\
\hline Cybianthus gardneri (A. DC.) G. Agostini & 2 & 0,30 & 0,18 & 0,49 \\
\hline Ouratea castaneifolia (DC.) Engl. & 2 & 0,30 & 0,12 & 0,42 \\
\hline Virola sebifera Aubl. & 2 & 0,30 & 0,08 & 0,38 \\
\hline Ilex affinis Gardner & 1 & 0,15 & 0,21 & 0,36 \\
\hline Byrsonima sp. & 2 & 0,30 & 0,05 & 0,35 \\
\hline Copaifera langsdorffii Desf. & 2 & 0,30 & 0,05 & 0,35 \\
\hline Tabebuia serratifolia (Vahl) G. Nicholson & 2 & 0,30 & 0,03 & 0,33 \\
\hline Galipea trifoliata Aubl. & 2 & 0,30 & 0,03 & 0,33 \\
\hline Abuta grandifolia (Mart.) Sandwith & 1 & 0,15 & 0,16 & 0,31 \\
\hline Hirtella racemosa Lam. & 1 & 0,15 & 0,10 & 0,25 \\
\hline Bellucia grossularioides (L.) Triana & 1 & 0,15 & 0,07 & 0,22 \\
\hline Indeterminada & 1 & 0,15 & 0,07 & 0,22 \\
\hline Vitex polygama Cham. & 1 & 0,15 & 0,06 & 0,21 \\
\hline Poecilanthe parviflora Benth. & 1 & 0,15 & 0,06 & 0,21 \\
\hline Roupala montana Aubl. & 1 & 0,15 & 0,04 & 0,19 \\
\hline Lonchocarpus campestris Mart. ex Benth. & 1 & 0,15 & 0,01 & 0,17 \\
\hline Qualea multiflora Mart. & 1 & 0,15 & 0,02 & 0,17 \\
\hline
\end{tabular}


Todas as árvores vivas

Sclerolobium paniculatum var. rubiginosum
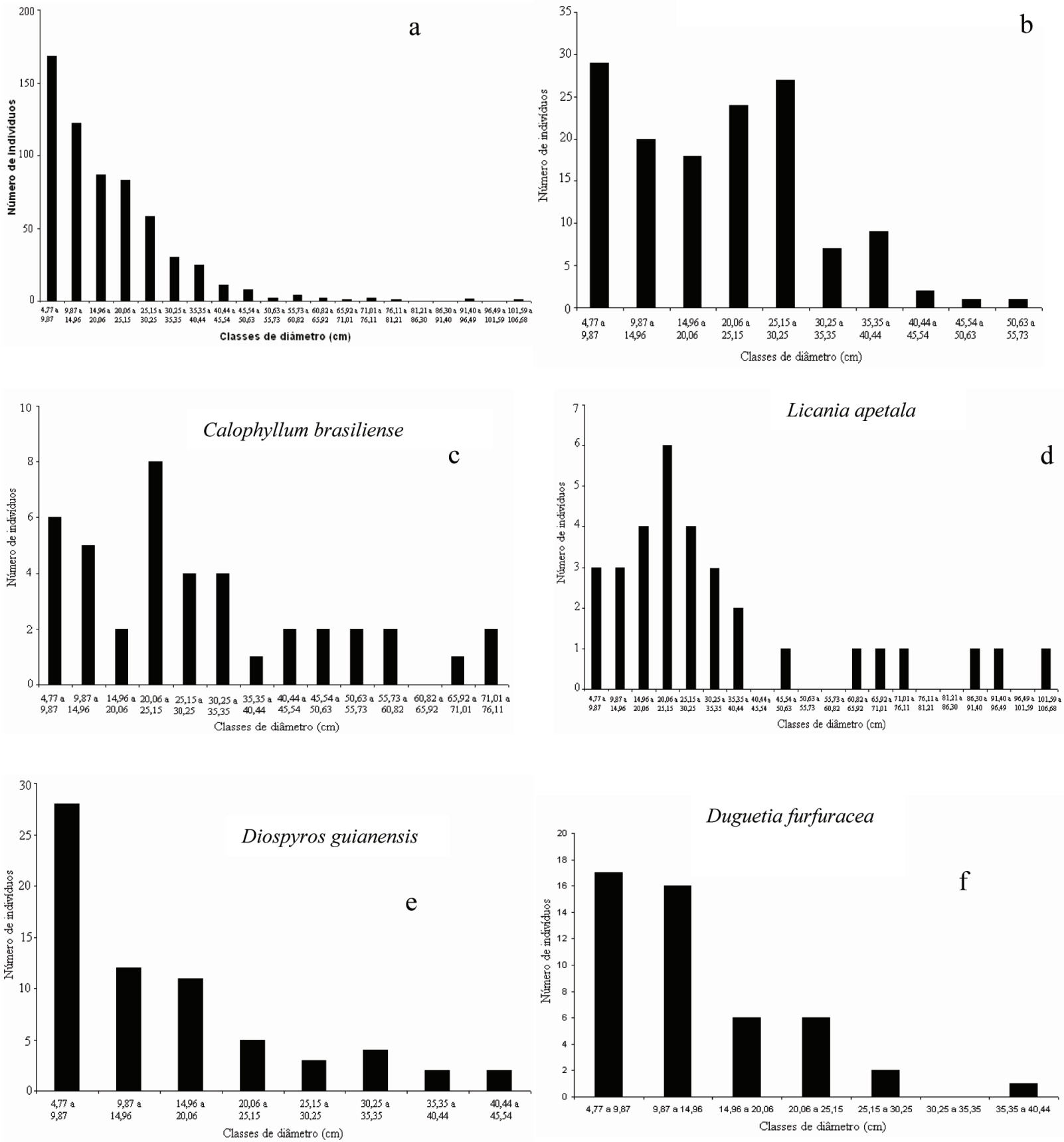

Figura 1 - Distribuição em classes de diâmetro, do total de indivíduos amostrados neste estudo e dos indivíduos das espécies de maior valor de cobertura. 


\section{BIBLIOGRAFIA CITADA}

Alvarez, V.H.; Novaes, R.F.; Barros, N.F.; Cantarut, R.B.; Lopes, A.S. 1999. Interpretação dos resultados das análises de solos. In: Ribeiro, A.C.; Guimarães, P.T.G.; Alvarez, V.H.(Eds.). Recomendaçôes para o uso de corretivos e fertilizantes em Minas Gerais: 5a aproximação. Viçosa: CFSEMG, p. 25-32.

APG II (ANGIOSPERM PHYLOGENY GROUP). 2003. An update of the Angiosperm Phylogeny Group classification for the orders and families of flowering plants: APG II. Botanical Journal of the Linnean Society, 141:399-436.

Bentes-Gama, M.M.; Scolforo, J.R.S.; Gama, J.R.V.; Oliveira, A.D. 2002. Estrutura e valoração de uma floresta de várzea alta na Amazônia. Cerne, 8(1):88-102.

Camargos, V.L. 2004. Floristica e estrutura da vegetação arbórea e suas relaçôes com a drenagem e os fatores edáficos num trecho de floresta estacional semidecidua no entorno da Lagoa Carioca Parque Estadual do Rio Doce-MG. Dissertação de Mestrado, Universidade Federal de Viçosa. Viçosa, Minas Gerais. 51p.

Cattanio, J.H.; Anderson, A.B.; Carvalho, M.S. 2002. Floristic composition and topographic variation in a tidal floodplain forest in the Amazon Estuary. Revista Brasileira de Botannica, 25:419-430.

Eiten, G. 1972. The cerrado vegetation of Brazil. Botanical Review, 38:201-341.

Eiten, G.1985. Vegetation near Santa Teresinha, NE Mato Grosso. Acta Amazonica, 15:275-301.

EMBRAPA - Empresa Brasileira de Pesquisa Agropecuária. 1997. Manual de métodos de análise de solo. Rio de Janeiro: EMBRAPA/ CNPS. 212p.

Felfili, J.M.; Silva Júnior, M.C. 1988. Distribuição dos diâmetros numa faixa de cerrado na fazenda Água Limpa (FAL), em Brasília-DF. Acta Botanica Brasilica, 2(1-2):85-105.

Felfili, J.M. 1994. Floristic composition and phytosociology of the gallery forest alongside the Gama stream in Brasília, DF, Brazil. Revista Brasileira de Botânica, 17:1-11.

Felfili, J.M.; Silva Junior, M.C.; Nogueira, P.E. 1998. Levantamento da vegetação arbórea na região de Nova Xavantina, MT. Boletim do Herbário Ezechias Paulo Heringer, 3:63-81.

Felfili, J. M.; Hilgbert, L. F.; Franco, A. C.; Silva, J.C.S.; Resende, A.V.; Nogueira, M.V.P. 1999. Behaviour of Sclerolobium paniculatum Vog. var. rubiginosum (Tul.) Benth. seedlings under different simulated shade intensities. Revista Brasileira de Botânica, 22:297-301.

Felfili, J.M.; Nogueira, P.E.; Silva Júnior, M.C.; Marimon, B.S.; Delitti, W.B.C. 2002. Composição florística e fitossociologia do Cerrado sentido restrito no município de Água Boa-MT. Acta Botanica Brasilica, 6(1):103-112.

Frangi, J.L.; Lugo, A.E. 1985. Ecosystem dynamics of a subtropical floodplain forest. Ecological Monographs, 55:351-369.

Guarim-Neto, G.; Morais, R.G. 2003. Recursos medicinais de espécies do cerrado de Mato Grosso: um estudo bibliográfico. Acta Botanica Brasilica, 17(4):561-584.

Harper, J.L.1977. Population biology of plants. London: Academic Press. 892 p.
Ivanauskas, N.M., Rodrigues, R.R.; Nave, A.G.1997. Aspectos ecológicos de um trecho de Florestas de Brejo em Itatinga, SP: florística, fitossociologia e seletividade de espécies. Revista Brasileira de Botânica, 20:139-153.

Lobo, P. C.; Joly, C. A. 2000. Aspectos ecofisiológicos da vegetação de mata ciliar do sudeste do Brasil. In: Rodrigues, R.R.; Leitão Filho, H.F. (Eds.). Matas ciliares: conservação e recuperação. São Paulo: Editora da Universidade de São Paulo: Fapesp. p.143 -157.

Martins, I. C. M., Soares, V. P., Silva, E.; Brites, R. S. 2002. Diagnóstico ambiental no contexto da paisagem de fragmentos florestais naturais "ipucas" - no Município de Lagoa da Confusão, Tocantins. Revista Árvore, 26:299-309.

Martins, S. V. 2001. Recuperação de Matas Ciliares. In: Martins, S. V.; Vieira, E. A. (Eds.).Viçosa: Aprenda fácil. 143 p.

Marques, M. C. M.; Joly, C. A. 2000. Estrutura e dinâmica de uma população de Calophyllum brasiliense Camb. em floresta higrófila do sudeste do Brasil. Revista Brasileira de Botânica, 23(1):107-112.

Marques, M.C.M.; Silva, S. M.; Salino, A. 2003. Florística e estrutura do componente arbustivo-arbóreo de uma floresta higrófila da bacia do rio Jacaré-Pepira, SP, Brasil. Acta Botanica Brasilica 17(4):495-506.

Mendonça, R. C.; Felfili, J.M.; Walter, B.M.T.; Silva Júnior, M.C.; Rezende, A.V.; Filgueiras, T.S.; Nogueira, P.E. 1998. Flora vascular do Cerrado. In: Sano, S. M.; Almeida, S. P.(Eds.) Cerrado, Ambiente e Flora. Planaltina. EMBRAPA/CPAC.. p. 289-556

Mileski, E. Aspecto da vegetação e do ecossistema da Ilha do bananal. Mapa fitoecológico e indicadores da pressão antrópica. Brasília: Secretária de Assuntos Estratégicos, 1994. $104 \mathrm{p}$.

Mueller Dombois, D.Y.; Ellenberg, M. 1974. Aims and methods in vegetation ecology. New York Willey and Sons, 547p.

Nascimento, M.T.; Saddi, N. 1992. Structure and floristic composition in an area of cerrado in Cuiabá-MT, Brazil. Revista Brasileira de Botânica, 15(1):47-55.

Nóbrega, M.G.G., Ramos, A. E.; Silva Júnior, M. C. 2001. Composição florística e estrutura na mata de galeria do Cabeçade-Veado no Jardim Botânico de Brasília - DF. Boletim do Herbário Ezechias Paulo Heringer, 8:44-65.

Oliveira-Filho, A.T. 1989. Composição florística e estrutura comunitária da floresta de galeria do Córrego da Paciência, Cuiabá (MT). Acta Botanica Brasilica, 3: 91-111.

Oliveira-Filho, A.T.1992. Floodplain "murundus" of Central Brazil: evidence for the termite-origin hypothesis. Journal of Tropical Ecology, 8(1):1-19.

Oliveira-Filho, A.T.; Ratter, J.A. 1995. A study of the origin of central Brazilian forests by the analysis of plant species distribution patterns. Edinburgh Journal of Botany 52(2):141-194.

Oliveira-Filho, A.T.; Fluminhan-Filho, M. 1999. Ecologia da vegetação do Parque Florestal Quedas do Rio Bonito. Cerne, 5(2)51-64. 
Oliveira-Filho, A.T.; Ratter, J. A. 2000. Padrões florísticos das matas ciliares da região do Cerrado e a evolução das paisagens do Brasil Central durante o quaternário tardio In: Rodrigues, R.R.; Leitão Filho, H.F. (Eds.). Matas ciliares: conservação e recuperação. São Paulo: Editora da Universidade de São Paulo. p.91-101.

Paschoal, M.E.S.; Cavassan, O. 1999. A flora arbórea da mata de brejo do ribeirão Pelintra, Agudos, SP. Naturalia, 24:172-191.

Pielou, E.C. 1975. The interpretation of ecological data. New York. John Wiley \& Sons, 263p

Ratter, J.A.; Ribeiro, J.F.; Bridgewater, S. 1997. The Brazilian cerrado vegetation and threats to its biodiversity. Annals of Botany, 80:230-233.

Ribeiro, R.J. Higuchi, N.; Santos, J.; Azevedo, C.P. 1999. Estudo fitossociológico nas regiōes de Carajás e Marabá - Pará, Brasil. Acta Amazonica, 29(2):207-222.

Scarano, F.R.; Ribeiro, K.T.; Moraes, L.F.D.; Lima, H.C. 1997. Plant establishment of flooded and unflooded patches of a freshwater swamp forest in southeastern Brazil. Journal Tropical Ecology, 14:793-803.

SEPLAN/TO. 2002. Atlas do Tocantins: Subsídios ao planejamento da gestão territorial. Secretaria do Planejamento e Meio Ambiente, Diretoria de Zoneamento Ecológico-EconômicoDZEE. 2ed. Palmas: Seplan, 49p.
Shepherd, G. J.1996. Fitopac 1: manual do usuário. Departamento de Botânica, UNICAMP, Campinas.

Silva Júnior, M.C. 1999. Composição florística, fitossociologia e estrutura diamétrica na mata de galeria do Monjolo, Reserva Ecológica do IBGE (RECOR), DF. Boletim do Herbário Ezechias Paulo Heringer, 4:30-45.

Toniato, M.T.Z., Leitão Filho, H.F.; Rodrigues, R.R. 1998. Fitossociologia de um remanescente de Floresta Higrófila (mata de brejo) em Campinas, SP. Revista Brasileira de Botânica, 21:197-210.

Torres, R.B., Mathes, L.A.F.; Rodrigues, R.R.; Leitão-Filho, H.F. 1992. Espécies florestais nativas para o plantio em áreas de brejo. O Agronômico, 44:13-16.

Torres, R.B., Mathes, L.A.F.; Rodrigues, R.R.1994. Florística e estrutura do componente arbóreo de mata de brejo em Campinas, SP. Revista Brasileira de Botânica, 17:189-194.

Recebido em 25/11/2004

Aceito em 08/07/2008 